\title{
Impact of entanglement density on solution electrospinning: a phenomenological model for fiber diameter
}

\author{
Chi Wang, ${ }^{* a}$ Yu Wang, ${ }^{a}$ Takeji Hashimoto ${ }^{* b}$
}

${ }^{a}$ Department of Chemical Engineering, National Cheng Kung University, Tainan 701, Taiwan, ROC

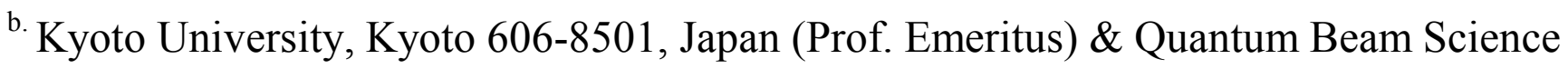
Center, Japan Atomic Energy Agency, Ibaraki 319-1195, Japan

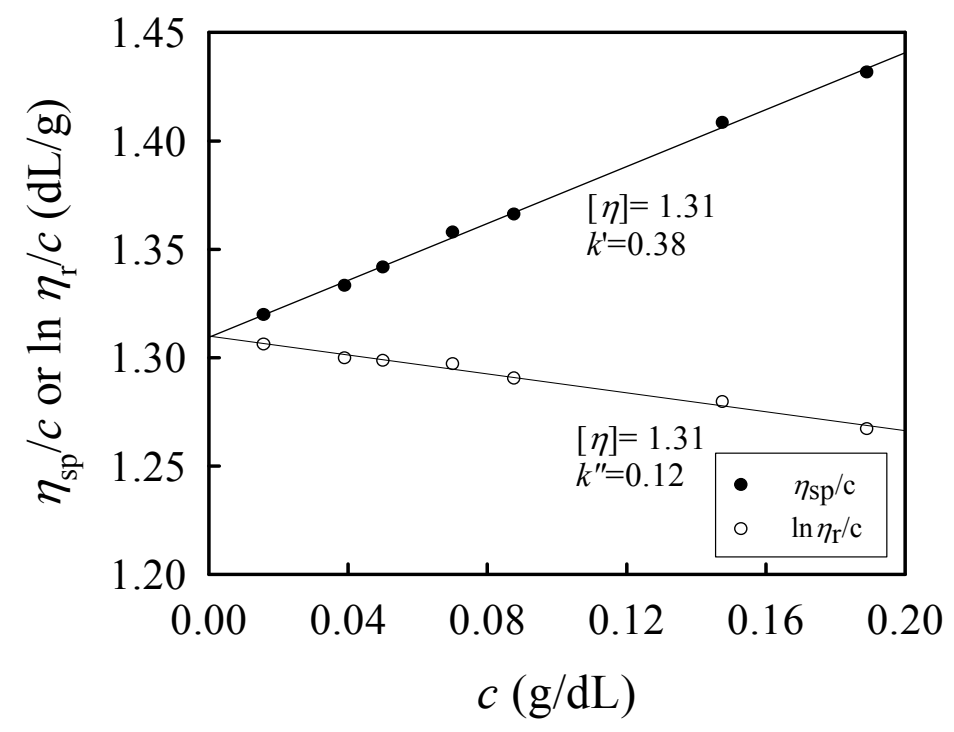

Figure S1. Determination of intrinsic viscosity of PNIPAM/DMF solution based on the Huggins equation, $\eta_{s p} / c=[\eta]+k^{\prime}[\eta]^{2} c$, and Kraemer equation, $\ln \eta_{r} / c=[\eta]-k^{\prime \prime}[\eta]^{2} c . \eta_{\text {sp }}$ and $\eta_{\mathrm{r}}$ are the specific viscosity and relative viscosity, respectively, and $c$ is the polymer concentration in the unit of $\mathrm{g} / \mathrm{dL}$. 

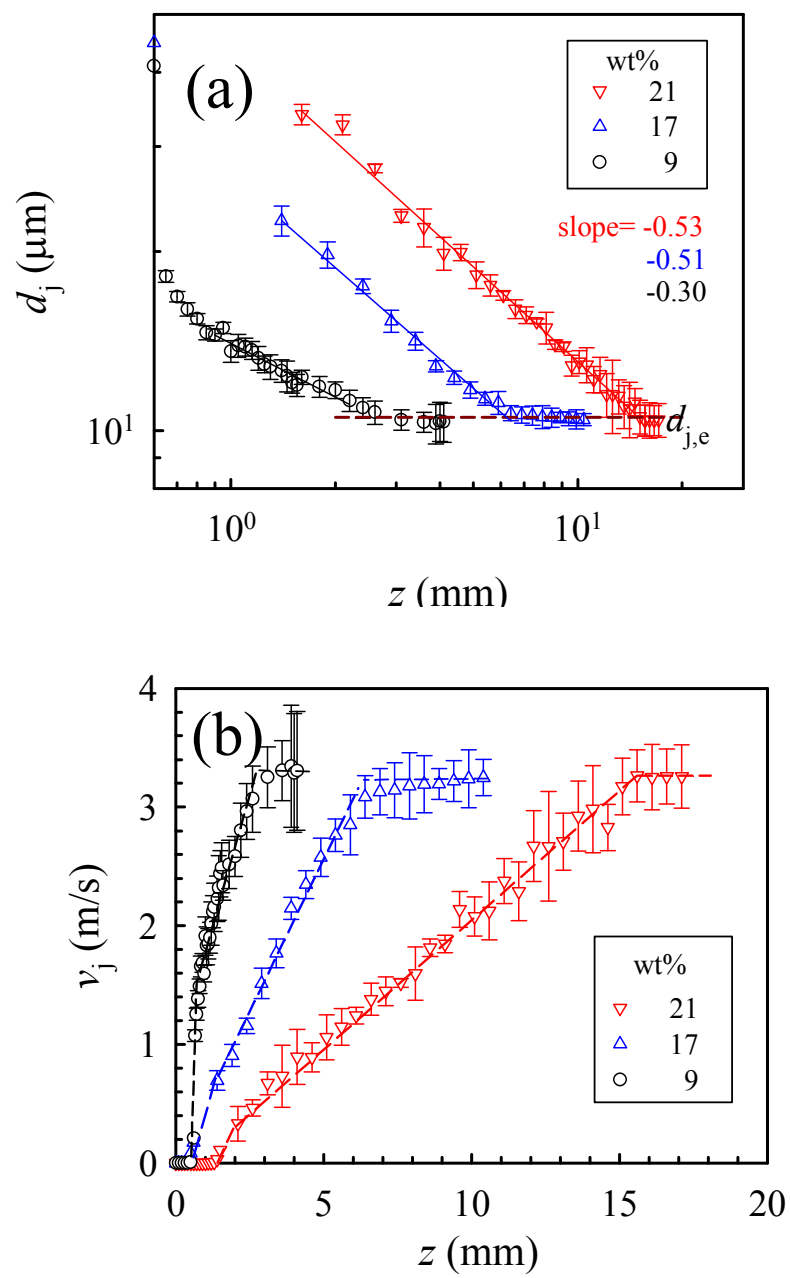

Figure S2. (a) Jet diameter profile along the jet length for electrospinning of PNIPAM/DMF solutions with different concentrations. A constant straight jet-end diameter, $d_{\mathrm{j}, \mathrm{e}}$, is seen, prior to the jet whipping process. (b) Calculated jet velocity profile. On the assumption that solvent evaporation is negligible, the jet velocity $V_{\mathrm{j}}$ is calculated by $4 Q / \pi d_{j}^{2}$. Then, the strain rate of jet $\varepsilon$ can be estimated by $\mathrm{d} V_{\mathrm{j}} / \mathrm{d} z$. The processing variables are: $1.0 \mathrm{~mL} / \mathrm{h}$, a voltage of $6.75 \mathrm{kV}$ and a tip-to-collector distance of $14 \mathrm{~cm}$. 

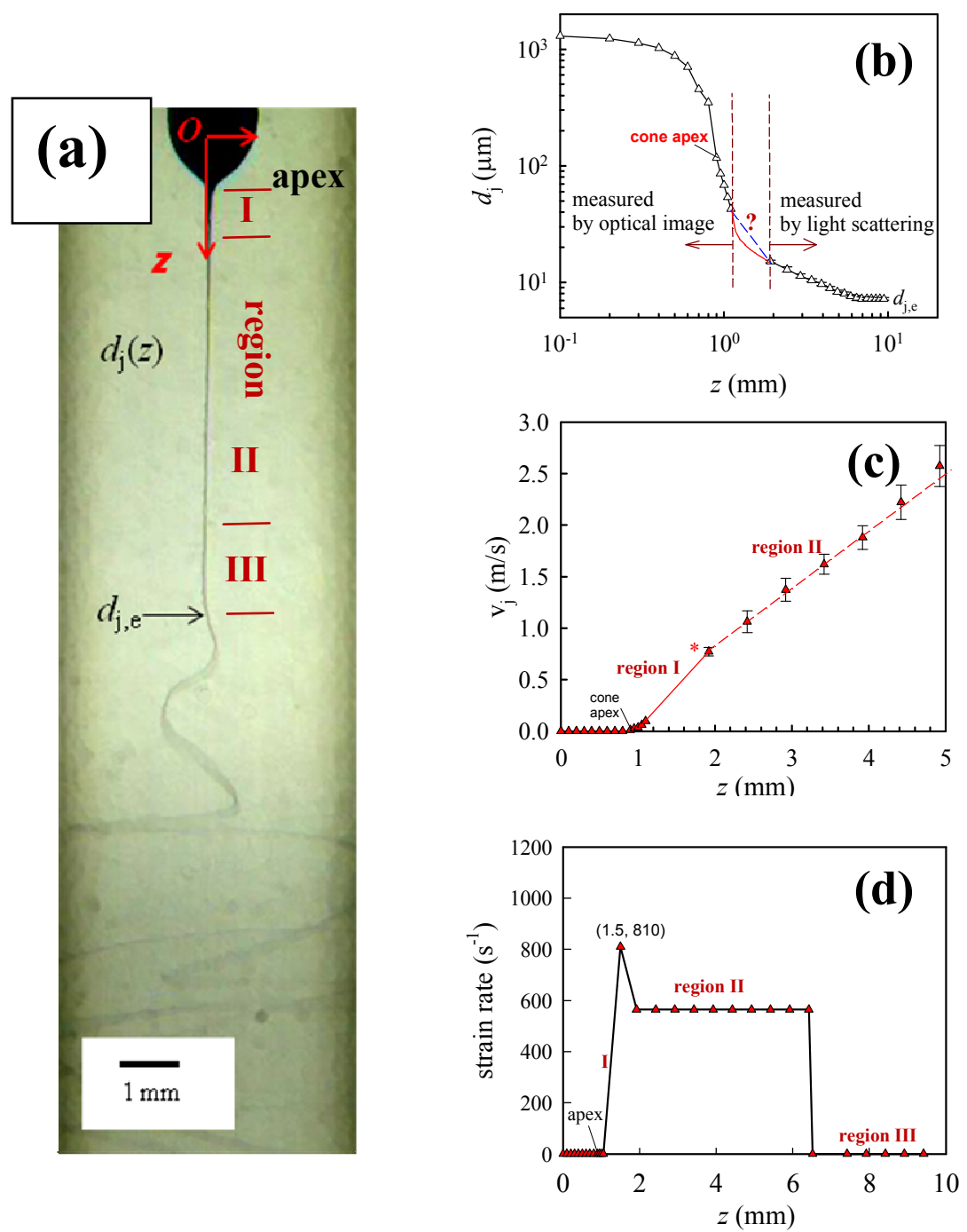

Figure S3. (a) High-speed camera image of $17 \mathrm{wt} \%$ solution during electrospinning. Three regions (I, II and III) for different strain rates are identified from the cone apex to the straight jet end, prior to jet whipping. The diameter of straight jet end is denoted as $d_{\mathrm{j}, \mathrm{e}}$. (b) The jet diameter is measured by analyzing the optical image for the $z$ ranging from 0 to $1.1 \mathrm{~mm}$, and by light scattering pattern for the $z$ ranging from 1.9 to $9.4 \mathrm{~mm}$. (c) The jet velocity profile derived under the mass conservation assumption. (d) The profile of strain rate along the straight jet.

Figure S3a shows the high speed camera image of electrospinning of $17 \mathrm{wt} \%$ PNIPAM/DMF solution. Along the $z$ axis, the cone size and jet diameter in the vicinity of cone apex are measured by the recorded optical images with a resolution of $15 \mu \mathrm{m}$ per pixel. Thus, reliable jet diameter of ca. 40 $\mu \mathrm{m}$ can be determined up to the $z$ value of $1.1 \mathrm{~mm}$. To obtain the jet diameter lower than $40 \mu \mathrm{m}$, the 
light scattering technique is feasible. However, the scattering pattern obtained from the vicinity of cone apex does not exhibit the intensity peak on the equator until $z$ is larger than $1.9 \mathrm{~mm}$. The absence of scattering maximum on the equator may suggest that the strain rate in this region is effectively larger than the Rouse relaxation rate. Provided that scattering peak is detected, its position is used to determined the $d_{\mathrm{j}}\left(=10.22 / q_{m, 1}\right)$. Thus, the jet diameter in the $z$ ranging from $1.1 \sim 1.9 \mathrm{~mm}$ cannot be resolved within the limitations of the present measuring apparatuses (Figure $\mathrm{S} 3 \mathrm{~b}$ ). Because of the limited data, the derived velocity profile exhibits a significant jump (Figure S3c) so that a discontinuity of the strain rate $\left(d v_{\mathrm{j}} / d z\right)$ is observed at $\mathrm{z}=1.9 \mathrm{~mm}$. The discontinuity gives rise to difficulties in determining the accurate strain rate in region I and the corresponding cumulative strain. Similar situations also occur to the other solutions with different concentrations. In Figure S3c, the slope in region I with $z$ ranging from 1.1 to $1.9 \mathrm{~mm}$ is tentatively derived to present the apparent strain rate of electrospinning jet in region $\mathrm{I}$, which is likely lower than the real value. Figure $\mathrm{S} 3 \mathrm{~d}$ shows the strain rate profile in different regions along the straight jet. Since the cumulative strain is strongly dependent upon the shape of velocity profile, its magnitude is subjected to some uncertainties as well.

Nevertheless, the following plots (Figures S3e, S3f, and S3g) illustrate the detailed evaluation of strain rates for the 15, 17 and $19 \mathrm{wt} \%$ solutions. The asterisk symbol marks the beginning of region II, at which the corresponding fluid time is set to zero. It is remarkable to notice that the traveling time in the region II is very short; ca. 1.7, 2.5 and $3.8 \mathrm{~ms}$ for the 15,17 and $19 \mathrm{wt} \% \%$ solutions, respectively. Moreover, the traveling time in region III for the 15,17 and 19 wt.\% solutions are $0.7,1.0$ and $0.6 \mathrm{~ms}$, respectively. In region II, the cumulative strain of the jet is correctly evaluated by $\int \varepsilon / v_{j} d z$; the calculated strain is $0.88,1.41$ and 1.63 for the 15,17 and 19 wt.\% solution, respectively. In region I, however, the cumulative strain may be uncertain due to the limited data for the jet diameter; the estimate strain is about $0.42,0.95$ and 0.79 for the 15,17 and 19 wt.\% solution, respectively. 

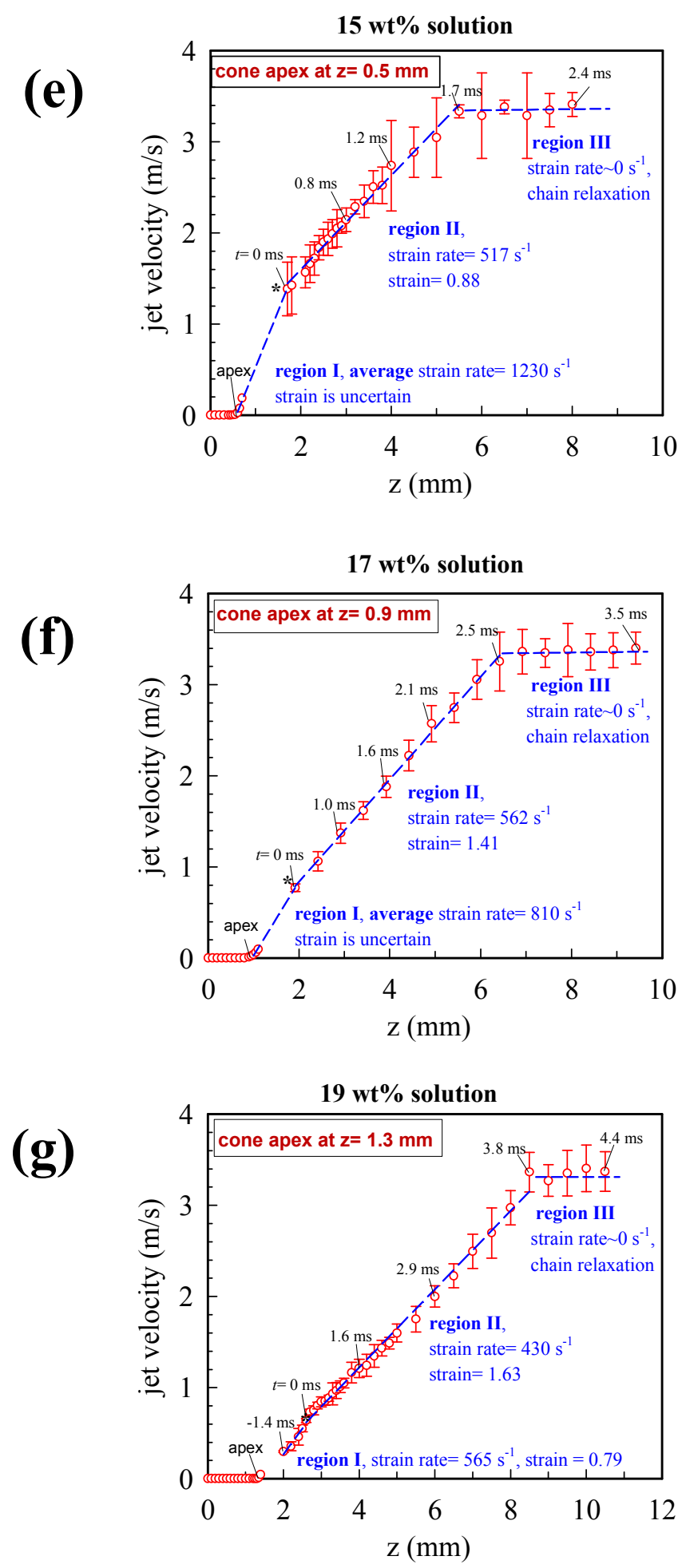

Figure S3. Velocity profile along the straight jet during electrsopinning of the (e) 15, (f) 17 and (g) 19 wt.\% solutions. The asterisk symbol marks the beginning of region II, at which the corresponding fluid time is set to zero. 


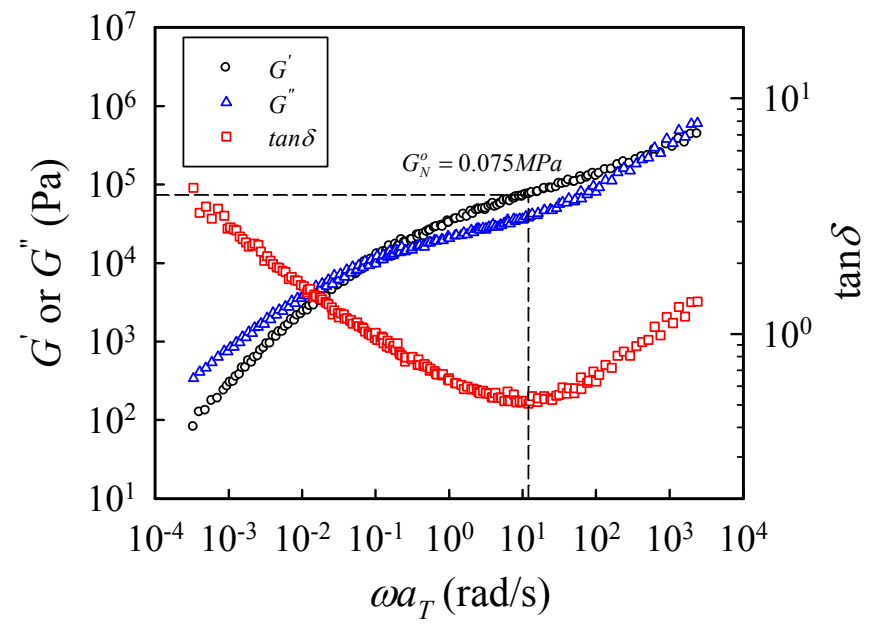

Figure S4. Since there was no reported value of $G_{N}^{o}(1)$ for the PNIPAM, we carried out the frequency sweep measurements of the undiluted PNIPAM melt at various temperatures $\left(170-250{ }^{\circ} \mathrm{C}\right)$. Based on the time-temperature superposition principle, the master curves of storage modulus $\left(G^{\prime}\right)$, loss modulus $\left(G^{\prime \prime}\right)$, and $\tan \delta\left(=G^{\prime \prime} / G^{\prime}\right)$ at a reference temperature of $190{ }^{\circ} \mathrm{C}$ are shown.

In principle, the best way to determine the plateau modulus $G_{N}^{o}(1)$ is to use narrow MWD and high MW samples, and to analyze the obtained $G$ "' $(\omega)$ curve by the 'integral method' (Liu et al., Polymer 2006, 47, 4461). However, no visual $G$ ' plateau and no visual $G$ " minimum are detected in Figure S4, suggesting that the molecular weight of our PNIPAM $\left(6.5 \times 10^{5} \mathrm{~g} / \mathrm{mol}\right)$ is not sufficiently high so that a severe overlap with high frequency Rouse modes is expected. Based on these facts, it is rather difficult, if not impossible, to completely extract the contribution of Rouse mode relaxation from the measured $G^{\prime}(\omega)$ and $G^{\prime \prime}(\omega)$ curves. According to the minimum $\tan \delta$ criterion, the $G^{\prime}$ value at the frequency where $\tan \delta$ reaches minimum is tentatively taken as $G_{N}^{o}(1)$. On this basis, the determined value of $G_{N}^{o}(1)$ is $0.075 \mathrm{MPa}$. The low $G_{N}^{o}(1)$ obtained is likely associated with the strong interaction between the amide side groups, which makes the backbone chains stiff. 
The density of PNIPAM at $25^{\circ} \mathrm{C}$ provided by the supplier is $1.1 \mathrm{~g} / \mathrm{cm}^{3}$. The glass transition temperature of PNIPAM is $130^{\circ} \mathrm{C}$ measured from the DSC heating scan at a rate of $10^{\circ} \mathrm{C} / \mathrm{min}$. According to the Simha-Boyer relations (J. Chem. Phys., 1962, 37, 1003), the magnitudes of $\alpha_{\mathrm{g}} T_{\mathrm{g}}$ and $\alpha_{\mathrm{L}} T_{\mathrm{g}}$ for amorphous polymers are 0.051 and 0.164 , respectively. $\alpha_{\mathrm{g}}$ and $\alpha_{\mathrm{L}}$ are the thermal volume expansion coefficients for polymers in the glass and melt states, respectively. Based on these results, the deduced density of PNIPAM melt at $190^{\circ} \mathrm{C}$ is ca. $1.058 \mathrm{~g} / \mathrm{cm}^{3}$. Thus, the determined value of $M_{\mathrm{e}}(1)$ is ca. $54,000 \mathrm{~g} / \mathrm{mol}$. 